\title{
COMPARISON OF GA-OPTIMIZED VISCOELASTIC MODELS FOR THE CHARACTERIZATION OF COMPRESSION BEHAVIOR OF WARP-KNITTED SPACER FABRICS
}

\author{
Masih Yousefpour, Mohammad Saleh Ahmadi*, Pedram Payvandy \\ Textile Engineering Department, Yazd University, 89168-69511 Yazd, Iran \\ ${ }^{*}$ Corresponding author. Email: ms.ahmadi@yazd.ac.ir
}

\begin{abstract}
:
Nowadays, Warp-Knitted Spacer Fabrics (WKSF) have been widely used for many technical applications. Compressional behavior of WKSF is one of their important properties. Physical modeling is one of the solutions to predict these properties for engineered designing of WKSF. In this study, four common physical models are introduced and compared in order to simulate compressional behavior of polyester WKSF. Genetic Algorithm (GA) was applied to optimize each model parameter. The results showed that the Burger model has the highest adoption with 0.2 percent Mean Absolut Error (MAE). The effect of thickness, outer fabric structure and spacer monofilament density on viscoelastic properties of the samples were also studied.
\end{abstract}

\section{Keywords:}

Compression behavior; warp-knitted spacer fabric; physical model; genetic algorithm; optimization

\section{Introduction}

Spacer fabrics include two outer layers that are joined together but kept apart by a middle layer of spacer monofilaments. [1] It is a known fact that spacer fabrics offer good impact and compression properties. The yarn loop will have large deformations when the knitted fabric is subjected to an impact or pressure loading.[2] Spacer yarn inclination angle and diameter, fabric thickness, outer layer structure, and yarn material properties can affect the impact and compression properties of warp-knitted spacer fabrics. Liu et al. studied the compression behavior of WKSF for cushioning applications. They investigated the effect of different structural parameters such as monofilaments inclination angle and fineness, fabric thickness and fabric layer structure. The results showed that lower monofilament inclination angle, higher fabric thickness, finer spacer yarn and larger size of surface structure meshes result in lower energy absorption in compression.[3] Du and $\mathrm{Hu}$ analyzed the spherical compression properties of WKSF theoretically. They investigated the deformation shape of the WKSF under spherical compression and established a relationship between total compression force and strain at the maximal compression point. The results showed that increasing the ball radius decreases the spherical compression effects.[4] Du and Hu (2012b), in another research, compared the experimental and theoretical data in maximal compression force, compression work and compression linear degree. The results showed about 7 percent difference between theoretical and experimental values.[5] Liu and Hu studied the effect of test boundary condition and sample size on the compression behavior of WKSF. They showed that the compression test boundary condition only affects the deformation behavior in the last stage of stress-strain curves (plateau stage). The sample size has an obvious effect on the results. The size of $10 \mathrm{~cm} \times 10$ $\mathrm{cm}$ showed the most stable results.[6] Chen et al. investigated compressive deformation and load of spacer monofilament in WKSF. By theoretical analysis of the shape deformation, they obtained critical bearing capacities at different stages during plane plate compression. The analysis results also indicated that it is better for the compressive displacement of the spacer monofilament to be in the range of the Restful Stage (third stage of compression process of the spacer monofilament) to ensure a good feeling for the wearer.[7]

Mass-spring models have been widely used by researchers for the modeling of textiles in several areas such as impact and damage properties of woven fabrics,[8] stitched seams behavior in repetitive extension and properties of recovery,[9] dynamic lateral compression properties of woven and knitted fabrics in low-force impact,[10] stress-relaxation forecast of woven fabrics containing elastane yarn,[11] bending behavior of hemp woven fabrics,[12] tensile behavior of woven fabrics,[13] viscoelastic behavior of ballistic fabrics,[14] creep behavior of plain woven fabrics,[15] creep behavior of carpet yarns,[16] drying shrinkage of plain knitted fabrics,[17] elongation behavior of nonwoven geotextile materials,[18] compression behavior of spacer fabrics,[19] tensile behavior of geotextiles with nonwoven materials[20] and drop-mass impact response of WKSF.[21]

In most of the aforementioned studies, the reason of choosing the studied models among various kinds of viscoelastic models is not obviously explained. To the best of our knowledge, no research has been published to investigate the accuracy of viscoelastic models for measuring the compression behavior of WKSF. There are four common models that best describes the compression behavior of WKSF, that is, Maxwell, Kelvin-Voigt, Standard linear and Burger. The main purpose of this study is 
to compare the ability of above-mentioned models in simulating the compression behavior of WKSF.

\section{Experimental}

\subsection{Warp-knitted spacer fabrics (WKSF)}

Six types of WKSF used in this work were prepared from a local producer. These fabrics had different thickness and structural parameters. All the WKSF were produced on a double-needlebar Raschel machine (Karl Mayer HDR-RD), equipped with six guide bars (GB1-GB6). Guide bars GB1, GB2, GB5 and GB6 knitted front and back outer layers of WKSF, while guide bars GB3 and GB4 carried the spacer yarns to connect the two outer layers together. Polyester monofilament yarns of $0.2 \mathrm{~mm}$ in diameter were used for the spacer monofilaments and polyester multifilament of $600 \mathrm{D} / 192 \mathrm{~F}$ for the outer layer yarns.

The samples were knitted with four different outer fabric structures, namely Chain + inlay $(C)$, Fine rhombic mesh $(F)$,
Large rhombic mesh $(\mathrm{L})$ and Hexagonal mesh $(\mathrm{H})$. They also had different thicknesses and spacer monofilament densities.

The specifications of the WKSF are listed in Tables 1. Figure 1 shows the structure of WKSF samples.

\subsection{Spherical compression test}

Spherical compression test on composite samples was conducted by the SDL Atlas Testometric-micro 350 universal testing machine equipped with a $250-\mathrm{kg}$ load cell. The specimen of size of $50 \mathrm{~mm} \times 50 \mathrm{~mm}$ was placed on a flat platen connected to the lower jaw. A polished steel ball $25.4 \pm$ $0.02 \mathrm{~mm}$ in diameter connected to the upper jaw was pressed against the specimen. The compression tests were conducted at a speed of $10 \mathrm{~mm} / \mathrm{min}$. Figure 2 shows a specimen under compression test. Five tests were carried out for each sample.

\subsection{Modeling}

Mass-spring model is one of the popular physical models. This model's most important features are implementation feasibility

Table 1. Specification of samples

\begin{tabular}{|c|c|c|c|c|c|c|}
\hline $\begin{array}{l}\text { Fabric } \\
\text { code }\end{array}$ & $\begin{array}{c}\text { Code of } \\
\text { outer layer's } \\
\text { structure }\end{array}$ & $\begin{array}{c}\text { Thickness } \\
\text { (mm) }\end{array}$ & $\begin{array}{l}\text { Fabric density } \\
\qquad\left(\mathrm{g} / \mathrm{m}^{2}\right)\end{array}$ & $\begin{array}{l}\text { Courses per } \\
\text { centimeter } \\
\text { (CPC) }\end{array}$ & $\begin{array}{l}\text { Wales per } \\
\text { centimeter } \\
\text { (WPC) }\end{array}$ & $\begin{array}{c}\text { Spacer yarn } \\
\text { density } \\
(\text { number/cm²) }\end{array}$ \\
\hline L20/30 & L & 20 & 987.41 & 6 & 2.5 & 30 \\
\hline L20/48 & L & 20 & 1132.11 & 6 & 4 & 48 \\
\hline $\mathrm{F} 25 / 60$ & $\mathrm{~F}$ & 25 & 1951.66 & 5 & 6 & 60 \\
\hline $\mathrm{H} 25 / 60$ & $\mathrm{H}$ & 25 & 1977.03 & 5 & 6 & 60 \\
\hline C30/55 & $C$ & 30 & 2335.38 & 5 & 5.5 & 55 \\
\hline C35/55 & $\mathrm{C}$ & 35 & 2722.22 & 5 & 5.5 & 55 \\
\hline
\end{tabular}

(a) J
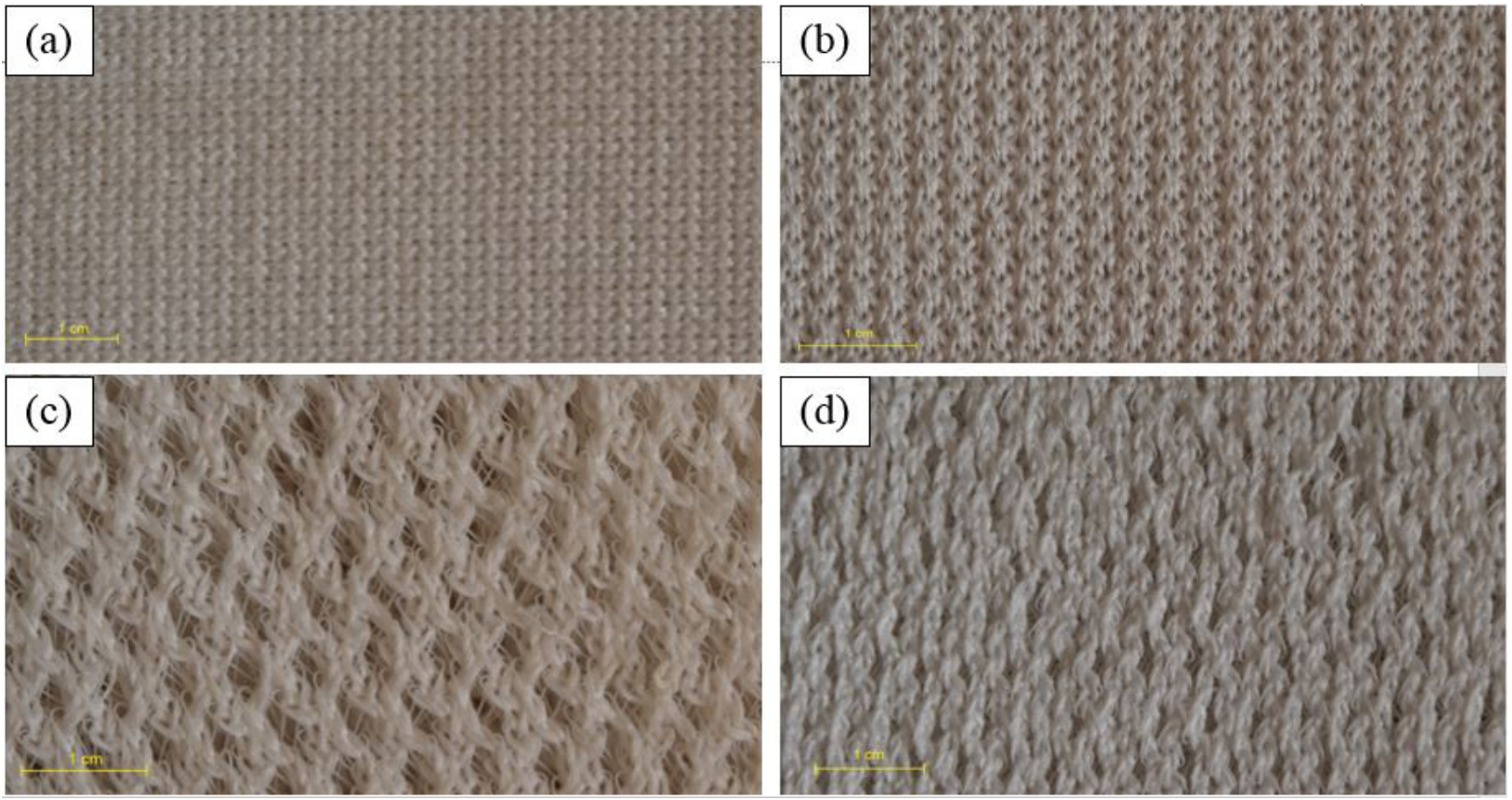

Figure 1. Warp knitted spacer fabric surface structures: a) Chain + inlay, b) Fine rhombic mesh, c) Large rhombic mesh, d) Hexagonal mesh 


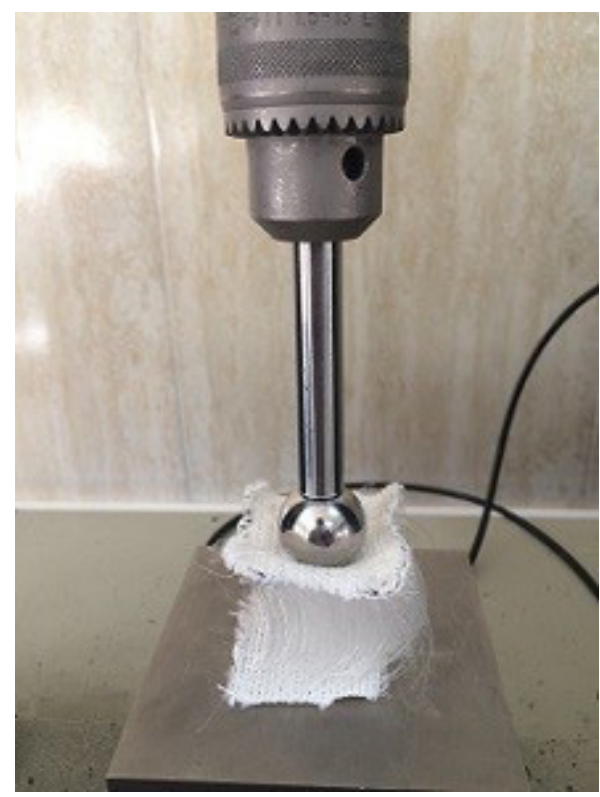

Figure 2. A specimen under compression test

and computation effectiveness. Therefore, mass-spring models are widely used in engineering modeling. Viscoelasticity is the property of materials that exhibits both viscous and elastic characteristics when undergoing deformation. The elastic components, can be modeled as springs and the viscous elastic components, can be modeled as dashpots. There are four popular kinds of viscoelastic models with different stressstrain behavior.

\subsubsection{Viscoelastic Maxwell model}

The Maxwell model can be represented by a purely viscous damper and a purely elastic spring connected in series (Figure 3a).

The model can be presented by the following equation:

$$
x(t)=F(t)\left(\frac{1}{k}+\frac{1}{c} t\right)
$$

where $\mathrm{k}$ and $\mathrm{c}$ are spring and dashpot coefficients, $\mathrm{F}$ is force $(\mathrm{N}), \mathrm{x}$ is displacement $(\mathrm{m})$ and $\mathrm{t}$ is time (s).

\subsubsection{Viscoelastic Kelvin-Voigt model}

The Kelvin-Voigt model, also known as the Voigt model, consists of a Newtonian damper and Hookean elastic spring connected in parallel (Figure $3 b$ ) with the following equation.

$$
x(t)=\frac{F(t)}{k}\left(1-\exp \left(-\frac{k}{c} t\right)\right)
$$

\subsubsection{Viscoelastic Standard linear model}

The Standard linear model effectively combines the Maxwell Model and a Hookean spring in series (Figure 3c) with the following equation.

$$
x(t)=F(t)\left(\frac{1}{k_{1}}+\frac{1}{k_{2}}\left(1-\exp \left(-\frac{k_{2}}{c} t\right)\right)\right.
$$

\subsubsection{Viscoelastic Burger model}

The Burger model provides a Kelvin-Voigt model acting in series with a Maxwell model, in both normal and shear direction (Figure $3 \mathrm{~d}$ ). The model can be presented by Equation (4):

$$
x(t)=F(t)\left(\frac{1}{k_{1}}+\frac{1}{c_{1}} t+\frac{1}{k_{2}}\left(1-\exp \left(-\frac{k_{2}}{c_{2}} t\right)\right)\right.
$$

\subsection{Genetic Algorithm (GA)}

The genetic algorithm (GA) is a computational model inspired by the biological evolution that can be used for optimization. This algorithm is based on the genetic manners of biological organisms. Population increases based on the principles of choice and existence of best that was reported by Charles Darwin. Following this method, the genetic algorithm can find a good result to linear and nonlinear problems by simultaneously exploring multiple regions of the result area and exponentially exploiting the promising spaces through mutation, crossover and selection operation. The basic principle of genetic algorithm

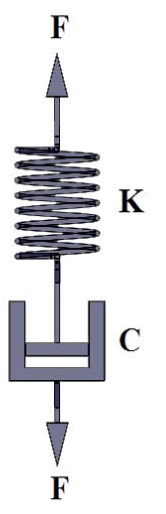

(a)

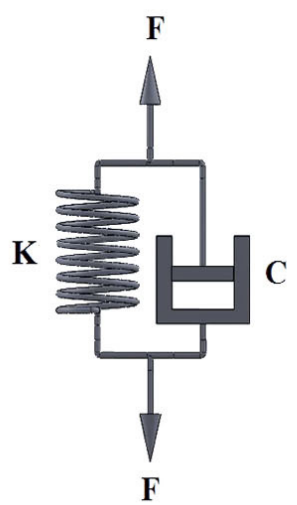

(b)

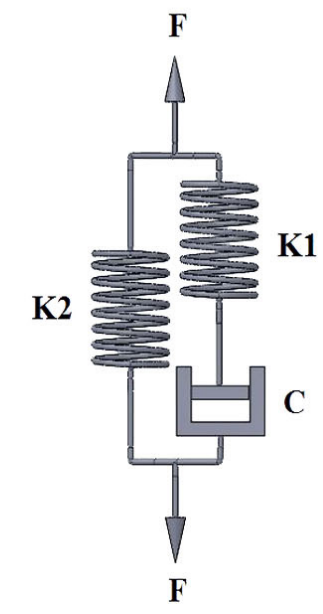

(c)

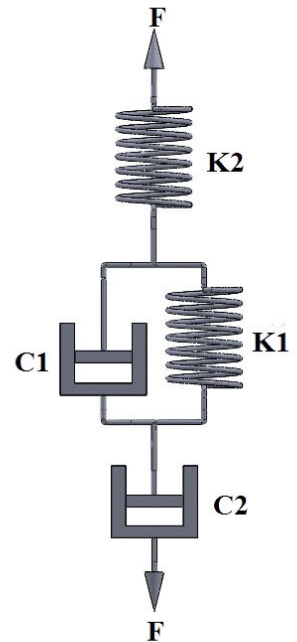

(d)

Figure 3. Four physical models: a) Maxwell, b) Kelvin-Voigt, c) Standard linear, d) Burger 
was first presented by John Holland in the 1960s.[22] Figure 4 shows the flowchart of a typical genetic algorithm.

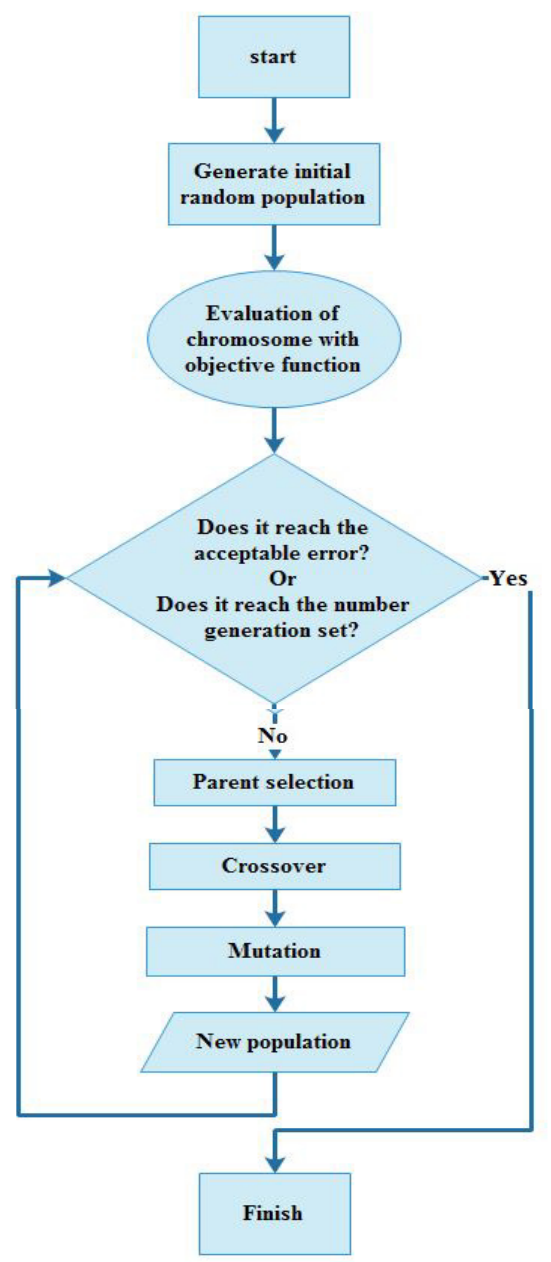

Figure 4. Genetic algorithm flowchart

\subsubsection{Determining the GA objective function}

The objective function evaluates the accuracy of the models' parameters. In GA, it is necessary that the difference between point positions obtained from the compression test data and the models get minimized. The less the difference, the closer the model's predictions to the WKSFs behavior. The general objective function can be defined by Equation (5).

$$
\text { Objective function }=\sum_{i=1}^{n}\left(\left|\left(X_{f i}-X_{m i}\right)\right| / n\right)(5)
$$

where $\mathrm{n}$ is the number of displacements, is the point position in the compression test and is the point position in the physical model referring to the Equations (1-4). Table 2 shows the GA parameters.

Table 2. Genetic algorithm parameters

\begin{tabular}{|c|c|}
\hline Parameters & Value/Property \\
\hline Population size & 1000 \\
\hline Number of generations & 100 \\
\hline Crossover percent & 0.8 \\
\hline Mutation percent & 0.3 \\
\hline Selection of parents & Roulette Wheel Selection \\
\hline Selection of crossover & Uniform \\
\hline
\end{tabular}

\section{Results and discussion}

Force-displacement curves of spherical compression test, which are based on the average of the five tests' results, are illustrated in Figure 5. It is seen that the highest compression force belongs to $F 25 / 60$ and $H 25 / 60$, which have the highest spacer monofilament densities. It shows that the density of spacer yarns (the number of yarns in the unit area of the sample) may have a considerable effect on the compression properties of the fabric. F25/60 has slightly higher compressional properties than $\mathrm{H} 25 / 60$. This may be attributed to the structures of their outer fabrics. F25/60 has a more dimensionally stable and less extensible structure than that of $\mathrm{H} 25 / 60$, so it shows slightly higher resistance against compression forces.

The coefficient of variations of the compression force for the samples at a specific displacement is given in Table 3 .

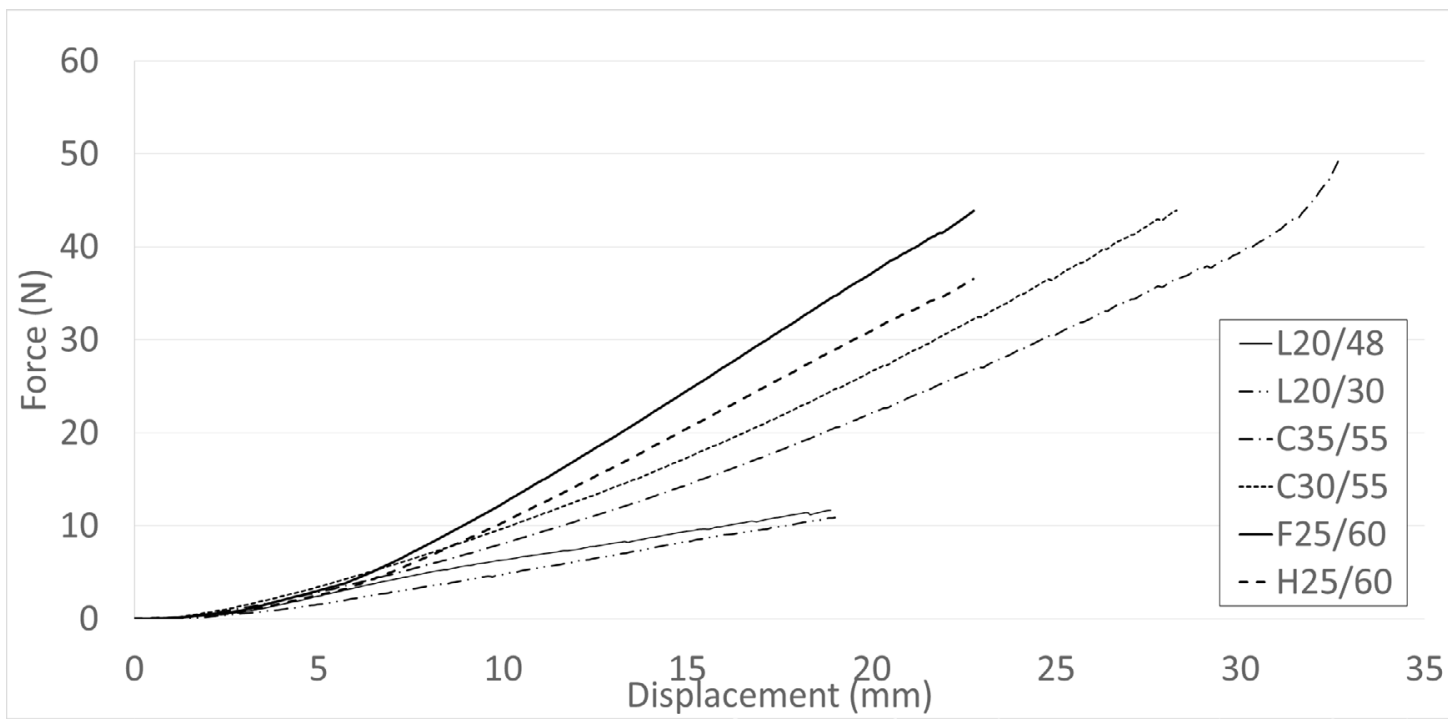

Figure 5. Results of spherical compression test 
Table 3. The coefficient of variations of the compression force for the samples

\begin{tabular}{|c|c|c|c|c|c|c|}
\hline Sample & L20/30 & L20/48 & C30/55 & C35/55 & H25/60 & F25/60 \\
\hline CV\% & 2.4 & 1.23 & 1.75 & 0.98 & 0.77 & 0.65 \\
\hline
\end{tabular}

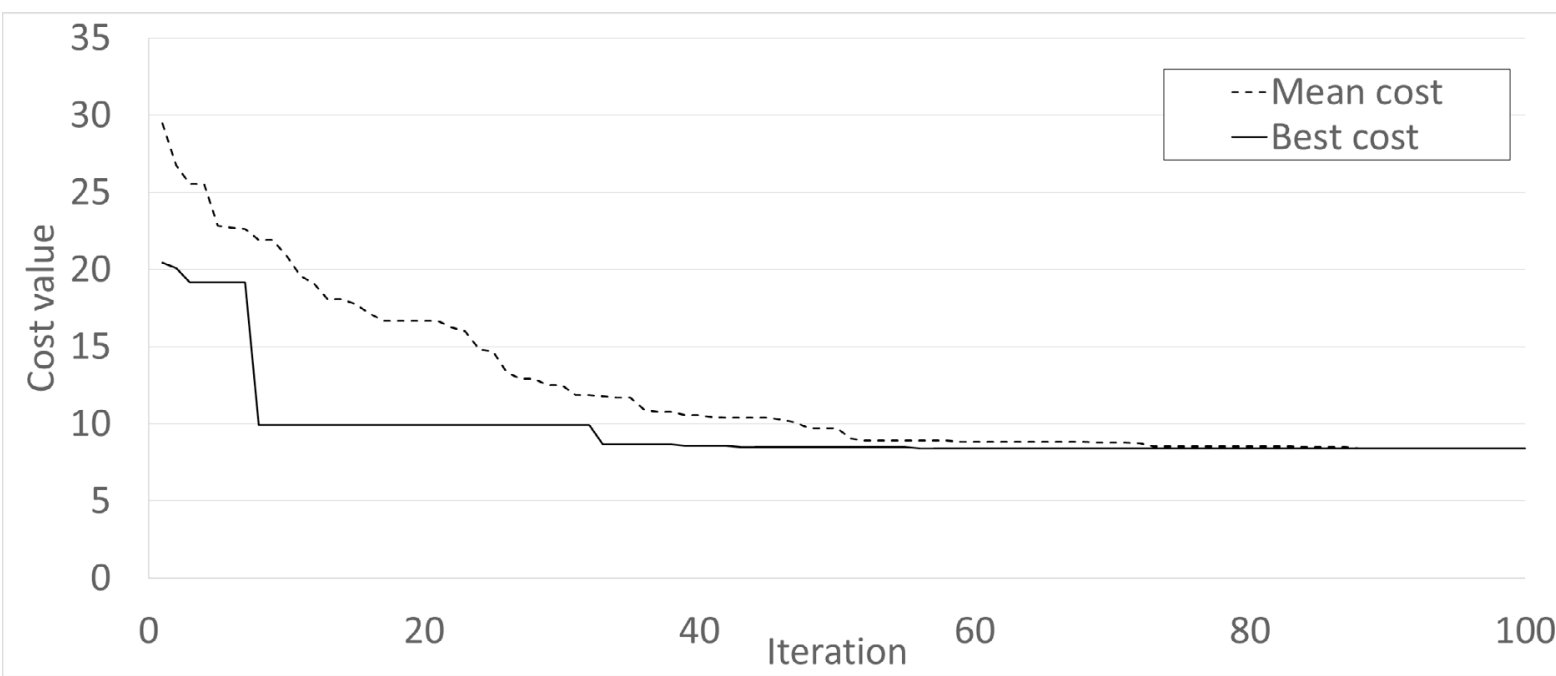

Figure 6. The best and mean cost of objective function for Maxwell model

Table 4. Optimal values and MAE of the models

\begin{tabular}{|c|c|c|c|c|c|c|c|c|c|c|c|c|c|c|c|}
\hline \multirow{2}{*}{ Sample } & \multicolumn{3}{|c|}{ Maxwell } & \multicolumn{4}{|c|}{ Kelvin-Voigt } & \multicolumn{3}{|c|}{ Standard linear } & \multirow[b]{2}{*}{ K1 } & \multicolumn{4}{|c|}{ Burger } \\
\hline & K & C & MAE & K & C & MAE & K1 & K2 & C & MAE & & K2 & C1 & C2 & MAE \\
\hline L20/30 & 152.2 & 2867.3 & 8.4 & 221.4 & 2967.8 & 5.5 & 210.8 & 0.5 & 2900.1 & 3.1 & 203.5 & 0.7 & 2846.3 & 161.4 & 0.4 \\
\hline L20/48 & 243.8 & 2255.1 & 7.3 & 391.3 & 2542.0 & 5.3 & 382.2 & 0.6 & 2425.7 & 2.3 & 325.0 & 0.8 & 2370.0 & 134.3 & 0.1 \\
\hline $\mathrm{F} 25 / 60$ & 430.2 & 3749.3 & 6.2 & 558.7 & 4001.5 & 5.1 & 520.4 & 1.1 & 3873.6 & 1.9 & 517.3 & 1.9 & 3698.0 & 197.6 & 0.2 \\
\hline $\mathrm{H} 25 / 60$ & 421.7 & 3798.4 & 6.2 & 520.6 & 4040.0 & 5.2 & 512.0 & 1.0 & 3905.6 & 2.2 & 497.7 & 1.8 & 3722.4 & 201.8 & 0.2 \\
\hline $\mathrm{C} 30 / 55$ & 332.2 & 4652.2 & 6.3 & 443.4 & 4918.0 & 5 & 421.2 & 0.6 & 4816.0 & 2.6 & 415.2 & 1.0 & 4713.3 & 324.2 & 0.4 \\
\hline C35/55 & 313.5 & 4930.9 & 7.6 & 437.0 & 5377.2 & 5.1 & 402.1 & 0.6 & 5257.5 & 2.1 & 398.3 & 0.9 & 5187.4 & 388.9 & 0.1 \\
\hline
\end{tabular}

The optimization was performed in Matlab 2015 software with a computer with following specifications: Corei7, 740Qm, $1.74 \mathrm{GHZ}$, and RAM $8 \mathrm{~GB}$. The mean execution time for optimization of the mechanism based on the GA was about 1 minute. Figure 6 shows the mean and the best costs for 100 GA iterations for L20/30 of Maxwell model. This figure clearly shows the convergence of the GA-based optimization process. By increasing the number of GA runs, the mean value of the objective function decreases and gradually approaches to the best value.

The best value for linear spring and dashpot coefficients were selected using GA with minimizing the objective function (Equation 5). After 10 runs of GA for each compression, the best parameters for the three aforementioned physical models were determined. The parameters are shown in Table 4.

According to Table 4, the Burger model has the minimum MAE $(0.2 \%)$. Figure 7 shows the Burger model and the real forcedisplacement curves in the model. MAE for Maxwell, Kelvin-
Voigt and Standard linear models were also 7, 5.2 and 2.3 percent, respectively. In fact, among the four common models compared in this study, the Burger model best describes the compression properties of WKSF.

F25/60 has the maximum spring coefficient and C35/55 has the maximum dashpot coefficient. It shows that monofilament density and thickness are important factors to determine spring and dashpot coefficients. In fact, increasing the number of spacer yarns in the unit area of the sample enhances its resiliency.

In the comparison of $F 25 / 60$ and $H 25 / 60$, one can see that F25/60 has higher spring coefficients and lower dashpot coefficients. In fact, the denser outer layer structure $(F)$ results in an increase in the spring property and a decrease in the dashpot property of WKSF. Comparing L20/30 and L20/48, with the same outer layer structures and thicknesses but different spacer yarn densities, it is seen that the sample with higher spacer yarn density has higher spring coefficient and 


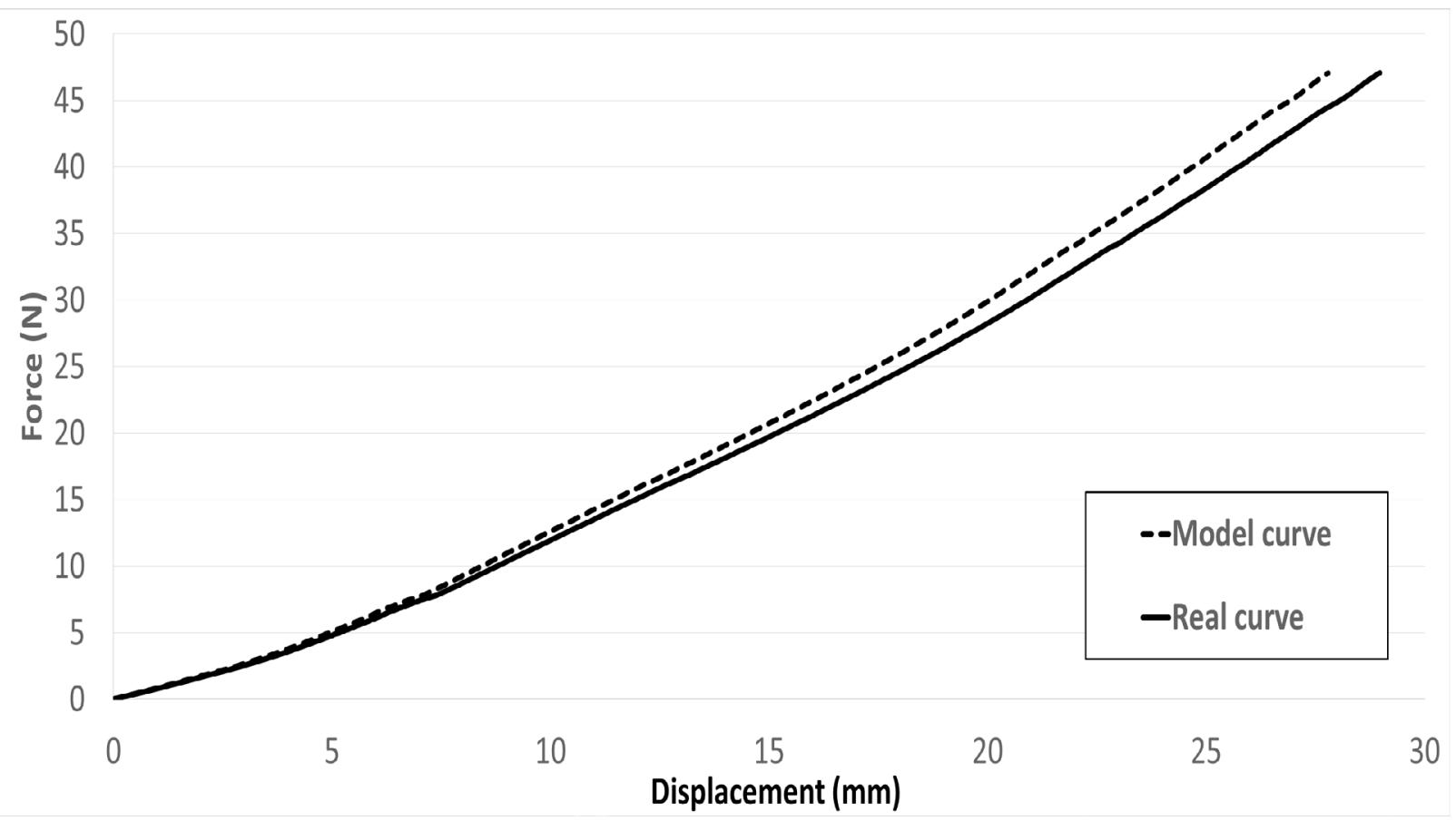

Figure 7. Force-displacement curves of modeling and real data (F25/60)

lower dashpot coefficient. Comparing C30/55 and C35/55, one can see that $\mathrm{C} 35 / 55$ with higher thickness has higher dashpot coefficient and lower spring coefficient. Therefore, increasing the thickness will result in enhancing the dashpot effect and declining the spring effect.

\section{Conclusion}

In this research, a comparative study was performed to assess the ability of four common physical models, that is, Maxwell, Kelvin-Voigt, Standard linear and Burger models, in characterizing the compression behavior of WKSF and the effect of WKSF parameters on spring and dashpot coefficients. For this purpose, GA was utilized for the determination of spring and dashpot coefficients. The results showed that the Burger model has maximum accuracy among the four physical models with a MAE of 0.2 percent. Spring and by increasing the monofilament density, surface fabric structure and dashpot coefficients increased by increasing the WKSF thickness. Also, WKSF thickness and surface fabric structure are more effective factors for the estimation of spring and dashpot coefficients in the compression behavior of WKSF. It was seen that the samples with higher thickness and denser outer fabric structure have lower spring and higher dashpot coefficients. Increasing the spacer monofilament density enhances the spring effect and reduces the dashpot effect of WKSF.

\section{References}

[1] Liu, Y., Hu, H., (2011). Compression property and air permeability of weft-knitted spacer fabrics. Journal of Textile Institute, 102, 366-372.

[2] Sun, B., Hu, D., Gu, B., (2009). Transverse impact damage and energy absorption of 3-D multi-structured knitted composite. Composites, 40, 572-583.
[3] Liu, Y., Hu, H., Zhao, L., Long, H., (2011). Compression behavior of warp-knitted spacer fabrics for cushioning applications. Textile Research Journal, 82, 11-20.

[4] Du, Z., Hu, H., (2012). A study of spherical compression properties of knitted spacer fabrics Part I: Theoretical analysis. Textile Research Journal, 85, 1569-1578.

[5] Du, Z., Hu, H., (2012). A study of spherical compression properties of knitted spacer fabrics part II: comparison with experiments. Textile Research Journal, 83, 794-799.

[6] Liu, Y., Hu, H., (2014). An experimental study of compression behavior of warp-knitted spacer fabric. Journal of Engineering Fiber and Fabric, 9, 61-69.

[7] Chen, M.-y., Lai, K., Sun, R.-j., Zhao, W.-z., Chen, X., (2016). Compressive deformation and load of a spacer filament in a warp-knitted spacer fabric. Textile Research Journal, 65, 200-211.

[8] Shim, V., Tan, V., Tay, T., (1995). Modelling deformation and damage characteristics of woven fabric under small projectile impact. International Journal of Impact Engineering, 16, 585-605.

[9] Webster, J., Laing, R., Enlow, R., (1998). Effects of Repeated Extension and Recovery on Selected Physical Properties of ISO-301 Stitched Seams Part II: Theoretical Model. Textile Research Journal, 68, 881-888.

[10] Taylor, P., Pollet, D., (2002). Low-force dynamic lateral compression of fabrics. Textile Research Journal, 72, 845-853.

[11] Šajn, D., Geršak, J., Flajs, R., (2006). Prediction of stress relaxation of fabrics with increased elasticity. Textile Research Journal, 76, 742-750.

[12] Mihailovic, T., (2006). Complex estimation of bending elasticity of hemp woven fabric after washing treatment. International Journal of Clothing Science Technology, 18, 70-82.

[13] Halleb, N., Ben Amar, S., (2008). Prediction of fabrics mechanical behaviour in uni-axial tension starting from their technical parameters. Journal of Textile Institute, 99, 525-532. 
[14] David, N., Gao, X.-L., Zheng, J., Masters, K., (2008). ThreeParameter Viscoelasticity Models for Ballistic Fabrics. in "the ASME 2008 International Mechanical Engineering Congress and Exposition, 459-466.

[15] Asayesh, A., Jeddi, A. A., (2010). Modeling the creep behavior of plain woven fabrics constructed from textured polyester yarn. Textile Research Journal, 80, 642-650.

[16] Gao, X., Sun, Y., Meng, Z., Sun, Z., (2012). Analytical approach of creep behavior of carpet yarn. Journal of Application Polymer Science, 124, 1160-1167.

[17] Mozafary, V., Payvandy, P., Jalili, M.M., (2014). Non-linear simulation of drying of plain knitted fabric using massspring-damper model and genetic algorithm optimization. International Journal of Advance Manufacturing Technology, 7, 67-76.

[18] Stepanovic, J., Stojiljkovic, D., Djordjic, D., Trajkovic, D., (2014). Elongation modeling of nonwoven geotextile materials/Modelarea alungirii materialelor geotextile netesute. Indian Texttile, 65, 90.
[19] Chattopadhyay, R., Kumar, B., Barik, P., (2015). Rheological model for compression of spacer fabrics. Fibers and Polymers, 16, 1554-1561.

[20] Stepanovic, J. M., Trajkovic, D., Stojiljkovic, D., Djordjic, D., (2015). Predicting the behavior of nonwoven geotextile materials made of polyester and polypropylene fibers. Textile Research Journal, 13, 1385-1397.

[21] Liu, Y., Hu, H., (2015). Compressive mechanics of warpknitted spacer fabrics. Part II: a dynamic model. Textile Research Journal, 85, 2020-2029.

[22] Lobo, F.G., Goldberg, D.E., (2004). The parameter-less genetic algorithm in practice. Information Sciences, 167, 217-232. 